\title{
DARK MATTER HALOS AROUND GALAXIES
}




\title{
DARK MATTER HALOS
}

\author{
R.P. SAGLIA \\ Institut für Astronomie und Astrophysik \\ Scheinerstraße 1, D-81679 München, Germany
}

\begin{abstract}
The observational evidence for dark halos around galaxies is shortly reviewed. New and old techniques and results constraining the mass, the distribution, the shape and the nature of dark halos are discussed.
\end{abstract}

\section{Introduction}

The casuality problem (why is the Cosmic Background Radiation so homogeneous, with temperature fluctuations $\Delta T / T \approx 10^{-5}$, Bennett et al. 1994 ?) and the fine-tuning of the density of the Universe (the present density $\Omega_{0}$ in units of the critical density should be near zero, if its initial value is different from 1 , Peebles 1993) force astronomers to believe in the inflation scenario (Guth, 1981), which produces a $\Omega=1$, flat Universe. At the same time, the constraints coming from the primordial nucleosynthesis of light elements require that at most $10 \%$ (Mathews et al., 1993) of this density can be in the form of baryons. Astronomers are therefore desperate for finding evidence of this remaining $90 \%$ dark component of the Universe.

This paper shortly summarizes the observational facts pointing to dark halos around galaxies and the properties of these halos. Comprehensive reviews of the subject are given by Carr (1994), Ashman (1992), Trimble (1987). The paper is organized as follows. Section 2 reviews the observational evidence for dark halos around galaxies, in the Milky Way (2.1), in spirals (2.2), in dwarfs (2.3), in ellipticals (2.4), in binary systems, galaxies with satellites or groups of galaxies (2.5), closing with the critical point of view of the "non-believers" (2.6). Sect. 3 summarizes the global properties of galaxy dark halos as derived from the observations: their tridimensional shape (3.1), their structure parameters and correlations (3.2), the constraints on their nature (3.3). Section 4 summarizes the conclusions and 
the future prospects.

\section{Observational Evidence}

\subsection{THE MILKY WAY}

Disk Dark Matter. The first attempt to find dark matter in galaxies is due to Oort $(1932,1960)$. Following his idea and using a sample of $\mathrm{F}$ dwarfs and K giants, Bahcall (1984) finds that the combined Poisson $\partial^{2} \Phi(z) / \partial z^{2}=$ $4 \pi G \rho$ and Boltzmann $\sigma_{z i}^{2} \partial \rho_{i}(z) / \partial z=-\rho_{i}(z) \partial \Phi(z) / \partial z$ equations required that at least $50 \%$ of the material in the disk has to be dark, confirming Oort's earlier result. Here $\Phi(z)$ is the gravitational potential in the solar neighborhood at a distance $z$ orthogonal from the disk and $\rho$ the disk density, while $\sigma_{z i}$ and $\rho_{i}$ are the velocity dispersions and the densities of stars of a given type $i$. However, more recent work (see Flynn \& Fuchs 1994 and references therein) tends to find a total dynamical surface-mass density in agreement with the visible surface density, without need for a dark component associated to the disk.

Halo Dark Matter. A dark halo is in any case needed, when an analysis similar to what done for external galaxies (see 2.2 and 2.5) is performed. Kochaneck (1995a) considers the Galaxy's (flat) rotation curve and the constraints coming from the local escape velocity of the stars, the motions of its satellites and the Local Group timing model. He derives a mass inside $50 \mathrm{kpc}$ between 3.3 and $6.1 \times 10^{11} M_{\odot}$ and a half-mass scale length of the halo between 110 and $300 \mathrm{kpc}$.

Baryonic Dark Matter. The most recent and exiting studies of the Milky Way dark halo come from the microlensing experiments. If the halo of the Galaxy is made of massive compact objects (MACHOs), by monitoring $\approx 10^{6}$ stars in the LMC, the SMC or the galactic bulge one should detect several achromatic luminosity enhancement events every year, caused by gravitational microlensing. The characteristic temporal dependence of the microlensing luminosity curve allows one to discriminate against variable stars and to constraint the masses of the microlensing objects. Comparing the rates of detections towards the different lines of sight, information about the MACHO halo shape can be gained. Following the ideas of Paczyński (1986), a number of collaborations started to search and find microlensing events: MACHO (Alcock et al., 1993), EROS (Aubourg et al., 1993), OGLE (Udalski et al., 1994). The rates derived up to now in the LMC direction rule out a dark halo made completely of MACHOs with masses between $8 \times 10^{-5}$ and $0.3 M_{\odot}$ but allow for $20 \%$ of the (spherical) halo to be made of MACHOs in the mass range $0.01-0.1 M_{\odot}$ (Alcock et al., 1995). Many more events than expected are instead derived towards the galactic bulge. This could imply that a new component of the Galaxy has been detected, 
such as a heavy disk or a bar, or that the galactic halo is more disk-like than spherical (Alcock et al., 1995). Finally, note that star counts in deep HST images rule out that a significant contribution to the mass of the halo comes from faint, hydrogen-burning stars (Bahcall et al., 1994).

\subsection{SPIRAL GALAXIES}

During the last 25 years the extended "flat" HI rotation curves of spiral galaxies have provided the best evidence for dark halos around extragalactic objects. Spiral galaxies are two-dimensional, rotationally supported and gas-rich stellar systems. The exponential radial decline of their surface brightness distribution should generate a keplerian $\left(\propto r^{-1 / 2}\right)$ rotation curve already at a few exponential scale lengths $h$ from the center. Rotational velocities are instead constant out to $\geq 11 h$ (NGC 3198, van Albada et al. 1985), thus requiring the presence of a probably spherical (see 3.1), isothermal-like (with density $\propto r^{-2}$ ) halo, with mass $\propto r$. Mass models constructed to fit the measured rotation curves try to minimize the amount of dark matter needed ("maximum-disk" solution) and produce the so-called "conspiracy problem" (van Albada \& Sancisi, 1986). The expected declining velocities of the disk component must be exactly compensated by the rising halo curves to give the nearly featureless flat data. At the same time, the constraint coming from the Tully-Fisher relation (Tully \& Fisher, 1977), linking the maximum rotational velocity to the luminosity of the galaxy, must be satisfied. Finally, a proper mass decomposition should also take into account the dynamical constraints coming from the observed presence of spiral structure (Athanassoula et al., 1987). Nowadays HI and optical rotation curves are available for more than a thousend galaxies (see 3.2). As a general result, the amount of dark mass needed to fit the rotation curves equals the luminous mass $M_{L}$ inside the optical limits $R_{\text {opt }}$ of the galaxies, and is $\approx 4 M_{L}$ inside $2 R_{\text {opt }}$, giving a typical total mass to blue luminosity ratio in the range $10-30 M_{\odot} / L_{\odot}$.

\subsection{DWARF GALAXIES}

Dwarf galaxies have low luminosity $\left(M_{B}>-17\right)$ and therefore a low baryonic content, offering the optimal "laboratory" to study the properties of dark matter halos. Rotation curves of gas-rich dwarf irregulars, analysed as described in 2.2 , give large dark to luminous mass ratios $(\approx 10)$ and imply large dark matter central densities (DDO 154, Carignan \& Freeman 1988; see also this book). Masses of (gas-free) dwarf spheroidals can be estimated from their luminosity core radii $r_{C}$ and their central stellar velocity dispersion $\sigma_{0}$, measured from repeated observations of radial velocities of single stars (Mateo, 1994). High dark matter central densities $\rho_{0}=9 \sigma_{0}^{2} /\left(4 \pi G r_{C}^{2}\right)$ 
and mass-to-light ratios $\left(\approx 100 M_{\odot} / L_{\odot}\right)$ are also derived here. These results rule out that the dark matter halos of dwarf galaxies are made of massive neutrinos. In this case, the present $\nu$ phase-space density should be less or equal than the one at the decoupling, implying either implausibly high neutrino masses or very short dynamical friction decay times (Gerhard \& Spergel, 1992a).

\subsection{ELLIPTICAL GALAXIES}

Until recently, no clear evidence for dark matter in ellipticals was available. These stellar systems are tridimensional, pressure supported and without cold gas, so that the dynamical information comes only from the analysis of their (difficult to measure) absorption line spectra. In addition, the interpretation of the so derived (projected) velocity and velocity dispersion profiles $\sigma(r)$ was affected by a degeneracy problem: not knowing the intrinsic distribution of stellar orbits, a flat $\sigma(r)$ profile (the analogous of the flat rotation curves of spirals) could have been interpreted as evidence for dark halos in the presence of radial or isotropic orbits, or as evidence for tangential orbits and constant $\mathrm{M} / \mathrm{L}$ ratio. Only in a couple of clear-cut cases (i.g. NGC 7144, Saglia et al. 1993) an increasing $\sigma$ profile rules out the tangential case. The analysis of the line of sight velocity distributions (LOSVD) in addition to $\sigma(r)$ offers now a general tool to break this degeneracy: tangential orbits produce flat-top LOSVDs, while radial orbits produce triangular shape LOSVDs (Bender et al., 1994). Jeske et al. (1995, also this book) apply this technique to the E0 galaxy NGC 6703 to constrain its gravitational potential and stellar orbital structure. A number of ellipticals (Saglia et al., 1995) are being analysed following this approach. Using similar data (Carollo et al. 1995) de Zeeuw and Carollo (this book) find evidence for dark halos in ellipticals using a stellar orbit superposition algorithm. Promising constraints on the extent of dark halos around ellipticals should come from the analysis of the radial velocity distributions of planetary nebulae (Arnaboldi et al., 1994) and globular clusters (Grillmair et al., 1994), although only with large amounts (> 500) of velocities the degeneracy due to the unknown orbital structure (analogous to what discussed above) can be disentangled.

Bright ellipticals have usually an X-ray corona, generated by thermal bremsstrahlung of a hot, massive gaseous component, in hydrostatic equilibrium in the gravitational potential of the galaxies (Fabricant et al., 1980). The gas density $\rho_{g}(r)$ and temperature $T_{g}(r)$ profiles can therefore be used to constrain the mass of the system as $M(r) \propto r T_{g}\left[d \log \rho_{g} / d \log r+\right.$ $\left.d \log T_{g} / d \log r\right]$. This approach allows one to probe the gravitational potential in the outer regions of ellipticals $\left(\leq 8 R_{e}\right)$, but suffers some severe 
uncertainties. Only recently temperature profiles have started to become available (Mushotzky et al., 1994) and it is difficult to estimate the role of the external pressure (Bertin et al., 1993). Evidence for dark halos around single ellipticals has also been gained when a gaseous ring or disk is present (Franx et al., 1994), or a gravitational lense is modeled (Kochanek, 1995b). Gravitational lensing statistics (Maoz \& Rix, 1993) requires also the presence of dark halos around early-type galaxies.

\subsection{BINARIES, SATELLITES, GROUPS}

The kinematics of binary galaxies, galaxies with companions or groups of galaxies give the opportunity to probe the typical total mass and scalelength of dark matter halos. Projected separations $r_{P}$ and relative velocities $\Delta v$ can be combined and averaged to estimate masses $M$ using the Virial Theorem: $M\left(3-2<e^{2}>\right)=32<r_{P} \Delta v^{2}>/(\pi G)$ (Zaritsky \& White, 1994), where $\left\langle e^{2}\right\rangle$ is the mean of the square ellipticity of the orbits. Mass estimates of single systems are however very uncertain, due to the problems caused by interlopers, the unknown orbital structure and the small number statistics. More stringent limits can be obtained, if models for halo formation are used to estimate these problems. Zaritsky \& White (1994) analyse a sample of 69 satellites using this approach and find a typical halo mass inside $200 \mathrm{kpc}$ of $1.5-2.6 \times 10^{12} M_{\odot}\left(H_{0}=75\right)$.

\subsection{THE OTHER POINT OF VIEW}

In the previous sections the observed dynamical discrepancies have been interpreted by invoking the presence of dark halos, using the newtonian laws. But also alternative interpretations have been proposed. Valentijn (1990) suggests that the luminous mass of the disks of spirals had been underestimated due to the presence of dust. More recent investigations (Peletier et al., 1995) seem to rule out this possibility. Lequeux (1994), Pfenniger et al. (1994) and Gerhard \& Spergel (1995, see also this book) argue that molecular hydrogen may have been strongly underestimated, especially in the outer regions of spiral galaxies. Finally, and more radically, Milgrom (1983) suggests MOND (modified newtonian dynamics) as the solution for the observed mass discrepancies. Accelerations in the low acceleration regime typical of the external regions of galaxy should tend to a (cosmic) constant, thus producing flat rotation curves. However, the dynamics of dwarfs (Gerhard \& Spergel, 1992b) and the existence of declining rotation curves (Persic et al., 1995) present strong challanges to this model. 


\section{Properties of Dark Halos}

\subsection{SHAPES}

Polar ring galaxies, disk galaxies with a gaseous ring orthogonal to the plane of the disk, offer the possibility to measure the velocity field in two perpendicular planes, and therefore to probe the gravitational potential and the dark halo shape. The analysis of the data has to involve the selfgravity contribution of the polar ring and in the best case studied, NGC 4650A, indicates a rather flattened (E6) halo ( Sackett et al., 1994a). A similar result (E5-E7) is found using a completely different technique, which compares the isophotes of optical and X-ray surface brightnesses of the elliptical NGC 720 (Buote \& Canizares, 1994). However, a rather round halo (E0.1-1.6) is needed to explain the twisted disk of NGC 4753 (SteimanCameron et al., 1992). Useful constrains should come from the analysis of the flaring of HI disks (Olling, 1995): very flattened halos should produce very little flaring and, therefore, could be ruled out. Inferring halo shapes from the modeling of warps may prove more dangerous (Dubinski \& Kujken et al., 1995).

Constraints on the axisymmetry of dark halos are much more stringent. The scatter that deviations from axisymmetry would induce on the TullyFisher relation (Franx \& de Zeeuw, 1992), the expansion of the HI velocity fields in harmonics (Franx et al., 1994), and the azimuthal structure of stellar disks in the infrared band (Rix \& Zaritsky, 1995) all imply that deviations from axisymmetry of the dark halos of galaxies are in the range E0-E0.6. Dissipationless dark matter N-body simulations generate instead rather strongly triaxial dark halos (Katz \& Gunn, 1991), which however may turn more axisymmetric when gas dynamics is included.

\subsection{GLOBAL CORRELATIONS}

The large data-base of rotation curves nowadays available (Persic et al., $1995)$ allows one to correct the simple picture of "flat rotation curves" of 2.2 and to investigate possible correlations of dark matter properties with the properties of galaxies. A luminosity dependence of the rotational velocities seems now well established, with low-luminosity galaxies showing rising rotation curves and high-luminosity galaxies having flat or declining curves (Persic et al. 1995, Casertano \& van Gorkom 1991). Equivalently, one finds that the dark to luminous mass ratio inside $R_{\text {opt }}$ scales with galaxy luminosity as $M_{D} / M_{L}\left(R_{\text {opt }}\right) \propto L_{B}^{-0.6}$.

A further step can be made by computing the core radii $r_{C}$, the central densities $\rho_{0}$ and velocity dispersions $\sigma_{0} \approx V_{\max } / \sqrt{2}$ of the dark halos found around dwarfs and late-type spirals, where the influence of the baryonic 
component should be minimal (see 2.2 and 2.3). Kormendy (1990) finds that dark matter halos in low luminosity galaxies are smaller, denser, and with lower velocity dispersion than those in high luminosity galaxies, with scaling laws similar to those obtained for the visible component.

\subsection{NATURE}

Are the dark halos around galaxies baryonic? Sackett et al. (1994b) detect a faint stellar halo around the edge-on galaxy NGC 5907, with a density profile able to reproduce the observed (flat) rotation curve, and suggest that faint stars could make the halos of galaxies. However, the limits coming from HST faint star counts exclude this possibility (see 2.1). The microlensing experiments suggest also that non hydrogen-burning, jupiter like compact objects cannot make all of the halo, but may be a fraction of it (see 2.1). Molecular hydrogen cloudlets (see 2.6) are still a possibility, although unlikely (Wilson \& Mauersberger, 1994).

Are the dark halos around galaxies non-baryonic? Massive neutrinos cannot make the halos of dwarfs (see 2.3) and therefore are not very appealing, However, a large number of weakly interacting massive particles (WIMPS) is "on the market", from axions to the lightest particle of supersymmetric theories ( Primack et al., 1988). Although yet undetected in the laboratory, WIMPS remain the best candidate constituents of dark matter halos, because of the successes of Cold Dark Matter type models (see Frenk, this book) in explaining the large scale structure of the Universe.

\section{Conclusions}

The existence of dark halos around spiral galaxies is well established, thanks to 25 years of measuring rotation curves. The next years of research should give us the same degree of confidence for the case of elliptical galaxies and assess the intrinsic shapes of dark halos, with gravitational lensing playing an important role. The steadily growing dynamical data-set should soon allow one to study the statistical properties of dark halos (for example, their mass function), and, possibly, their evolution with redshift (see Franx and Rix, this book). Hopefully, particle physicists will in the mean time detect the particles that constitute the dark matter.

Acknowledgments Financial support by the Deutsche Forschungsgemeinschaft under SFB 375 is gratefully acknowledged.

\section{References}

Alcock, C., et al. 1993, Nature 365, 621

Alcock, C., et al. 1995, preprint 
Arnaboldi, M., Freeman, K.C., Hui, X., Capaccioli, M., Ford, H. 1994, The Messenger 76, 40

Ashman, K.M. 1992, PASP 104, 1109

Aubourg, E. et al. 1993, Nature 365, 623

Athanassoula, E., Bosma, A., Papaioannou, S. 1987, A\&A 179, 23

Bahcall, J.N. 1984, ApJ 276, 169

Bahcall, J.N., Flynn, C., Gould, A., Kirhakos, S. 1994, ApJ 435, L51

Bennett, C.L., et al. 1994, ApJ 436, 423

Bender, R., Saglia, R.P., Gerhard, O. 1994, MNRAS 269, 785

Bertin, G., Pignatelli, E., Saglia, R.P. 1993, A\&A 271, 381

Buote, D.A., Canizares, C.R. 1994, ApJ 427, 86

Carignan, C., Freeman, K.C. 1988, ApJ 332, L33

Carollo, C.M., de Zeeuw, P.T., van der Marel, R.P., Danziger, I.J.,.Qian, E.E. 1995, ApJ 441, L25

Carr, B. 1994, ARAA 32, 531

Casertano, S., van Gorkom, J.H 1991, AJ 101, 1231

Dubinski, J., Kuijken, K. 1995, ApJ 442, 492

Fabricant, D., Lecar, M., Gorenstein, P. 1980, ApJ 241, 552

Flynn, C., Fuchs, B. 1994, MNRAS 270, 471

Franx, M., de Zeeuw, T. 1992, ApJ 392, L47

Franx, M., van Gorkom, J.H., de Zeeuw, T. 1994, ApJ 436, 642

Gerhard, O.E., Spergel, D.N. 1992a, ApJ 397, 38

Gerhard, O.E., Spergel, D.N. 1992b, ApJ 397, 38

Gerhard, O.E., Spergel, D.N. 1995, ApJL, in press

Grillmair, C.J., Freeman, K.C., Bicknell, G.V., Carter, D., Couch, W.J., Sommer-Larsen, J., Taylor, K. 1994, ApJ 422, L9

Guth, A. 1981, Phys. Rev. D23, 347

Jeske, G., Gerhard, O., Saglia, R.P., Bender, R. 1995, MNRAS submitted

Katz, N., Gunn, J.E. 1991, ApJ 368, 325

Kochanek, C.S. 1995a, preprint

Kochanek, C.S. 1995b, ApJ 445, 559

Kormendy, J. 1990, ASP 10, 33

Lequeux, J., 1994, A\&A 287, 368

Maoz, D., Rix, H-W. 1993, ApJ 416, 425

Mateo M. 1994, ESO/OHP Workshop on Dwarf Galaxies, B. Bingelli, G. Meylan Eds., ESO, Garching, p. 209

Mathews, G.J., Schramm D.N., Meyer, B.S. 1993, ApJ 404, 476

Mushotzky, R.F., Loewenstein, M., Awaki, H., Makishima, K., Matsuchita, K., Matsumoto, H. 1994, ApJ 436, L79

Milgrom, M. 1983, ApJ 260, 365

Olling R.P. 1995, AJ in press

Oort, J.H. 1932, Bull. Astron. Inst. Netherlands 6, 249

Oort, J.H. 1960, Bull. Astron. Inst. Netherlands 15, 45

Paczyński, B. 1986, ApJ 304, 1

Peebles, P.J.E. 1993 Principles of Physical Cosmology, Princeton University Press, Princeton

Peletier, R.F., Valentijn, E.A., Moorwood, A.F.M., Freudling, W., Knapen, J.H., Beckman, J.E. 1995, A\&A 300, L1

Persic, M., Salucci, P., Stel, F. 1995, MNRAS submitted

Pfenniger, D., Combes, F., Martinet, L. 1994, A\&A 285, 79

Primack, J.R., Seckel, D., Sadoulet, B. 1988, Ann. Rev. Nucl. Part. Sci. B38, 751

Rix, H-W., Zaritsky, D. 1995, ApJ 447, 82

Sackett, P.D., Rix, H.-W., Jarvis, B.J., Freeman, K.C. 1994a, ApJ 436, 629

Sackett, P.D., Morrison, H.L., Harding, P., Boroson, T.A. 1994b, Nature 370, 441

Saglia, R.P., et al. 1993, ApJ 403, 567 
Saglia, R.P., Bender, R., Jeske, G., Gerhard, O. 1995, in preparation

Steiman-Cameron, T.Y., Kormendy, J., Durisen, R.H. 1992, AJ 104, 1339

Trimble, V. 1987, ARAA 25, 425

Tully, R.B., Fisher, J.R. 1977, A\&A 54, 661

Udalski, A., Szymański, M., Kaluzny, J., Kubjak, M., Mateo, M., Krzemiński, W. 1994, ApJ 426, L69

Valentijn, E.A., 1990, Nature 346, 153

van Albada, T.S., Bahcall, J., Begeman, K., Sancisi, R. 1985, ApJ 295, 305

van Albada, T.S., Sancisi, R. 1986 Phil. Trans. Roy. Soc. London A 320, 447

Wilson T.L., Mauersberger R., 1994, A\&A 282, L41

Zaritsky, D., White, S.D.M 1994, ApJ 435, 599

\section{Discussion}

Fabbiano: Is there an explanation for the luminosity dependence of the rotation curve of spirals? I find it especially difficult to understand if the DM is non-baryonic.

Saglia: This is indeed a problem of CDM-like models. Navarro (this book) discusses the difficulties emerging when such a luminosity dependence is modeled.

Peletier: You say that measurements of the local mass density by Bahcall et al. imply that halos cannot be made out of faint stars. What about the possible detection by Morrison et al. (1994, AJ 108, 1191) and the modeling of Sackett et al. (1994b) of an optical halo in NGC 5907?

Saglia: see discussion in Sect. 3.3.

Faber: can you comment on evidence from X-rays around elliptical galaxies? Saglia: see discussion in Sect. 2.4.

Dejonghe: I would like to remind you of the case of NGC 7144 (Saglia et al., 1993), which has a rising velocity dispersion profile up to $2 R_{e}$. I do not know of any dynamical model, irrespective of dynamical structure and/or presence of LOSVDs, that can reproduce this without dark matter.

Saglia: I agree with you. However, this is a single case. The method described in 2.4 allows us to study the problem of dark matter in all ellipticals.

Dehnen: From the theories of early density perturbations (in good agreement with $\mathrm{COBE}$ ) we expect the (dark) matter to have power on all scales, especially on those smaller than can be resolved by current simulations of structure formation in the universe. This implies that dark matter halos must also have cusps. Is there any evidence in this direction?

Saglia: Dark halos of CDM-like models without gas dynamics do not reproduce the density profiles of observed dark halos, see discussion by Burkert and Navarro in this book. 


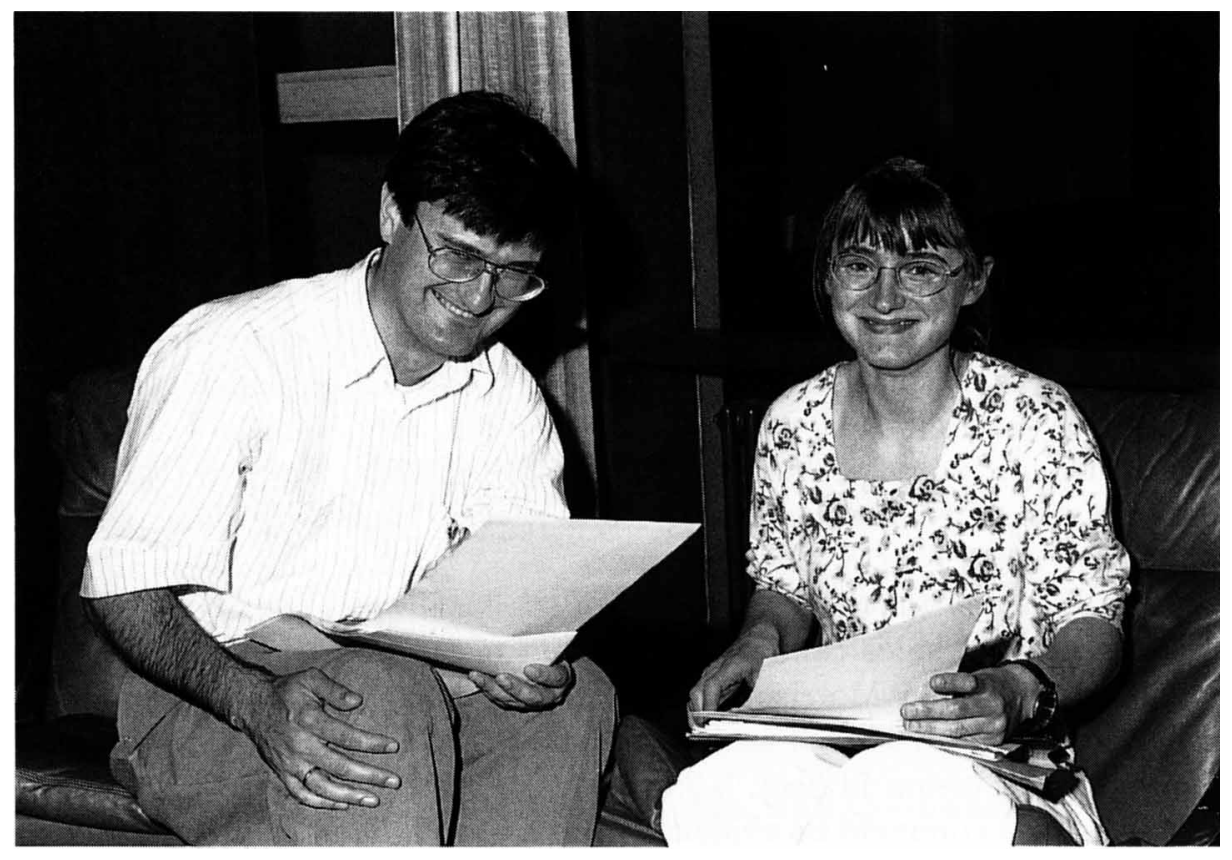

Roberto Saglia and Claire Halliday 